\title{
Drug Susceptibility Profile of Mycobacterium tuberculosis Isolated from Patients Visiting National Tuberculosis Centre, Nepal
}

\author{
Sangita Dahal ${ }^{1}$, Megha Raj Banjara ${ }^{1}$, Dhruba Kumar Khadka ${ }^{2}$, Gokarna Raj Ghimire ${ }^{2}$, Supriya \\ Sharma ${ }^{1 *}$ \\ ${ }^{1}$ Central Department of Microbiology, Tribhuvan University, Kirtipur, Kathmandu, Nepal \\ ${ }^{2}$ National Tuberculosis Centre, Thimi, Bhaktapur, Nepal
}

*Corresponding author: Ms. Supriya Sharma, Central Department of Microbiology, Tribhuvan University, Kirtipur, Kathmandu, Nepal; E-mail: suprisharma@hotmail.com

\begin{abstract}
Objectives: The objective of this study was to assess drug susceptibility pattern of Mycobacterium tuberculosis (MTB).

Methods: This cross-sectional study was carried out among 145 clinically suspected and previously treated pulmonary tuberculosis patients visiting National Tuberculosis Centre, Bhaktapur, Nepal. After obtaining written informed consent, questionnaire was administered and sputum samples were collected from each patient. Each sample was subjected to Ziehl-Neelsen (ZN) staining and cultured on Lowenstein Jensen (LJ) medium at $37^{\circ} \mathrm{C}$ for 8 weeks. MTB isolates were identified by growth rate and colony morphology, confirmed by biochemical tests. Drug susceptibility testing (DST) of identified isolates was performed by proportion method.
\end{abstract}

Results: A total of $49.7 \%(n=72)$ sputum samples were positive for MTB by culture and $46.9 \%(n=68)$ were positive by ZN staining. Among culture positive isolates of MTB ( $n=72), 25 \%(n=18)$ were resistant to at least one drug. The prevalence of multi drug resistant tuberculosis (MDR-TB) was $15.3 \%(n=11)$ of which $5.5 \%(n=4)$ were resistant to rifampicin (RIF) only, $1.3 \%(n=1)$ were resistant to isoniazid (INH) only. Out of 18 resistant isolates, $61.1 \%(n=11)$ were resistant to both RIF and INH, $21.4 \%(n=3)$ resistant to INH were susceptible to RIF and $26.6 \%(n=4)$ resistant to RIF were susceptible to INH. Smoking $(\mathrm{P}=0.001)$ and coughing $(\mathrm{P}=0.009)$ were statistically significant with isolation of MTB.

Conclusion: Since the prevalence of MDR-TB was high, MDR-TB strains should be identified in order to initiate second line treatment.

Key words: TB, MDR-TB, Smoking, Nepal

\section{INTRODUCTION}

Tuberculosis (TB) is a ninth leading cause of death by an infectious disease worldwide, despite global efforts and financial investments by governments and nongovernmental organizations in disease-control programs during the past 20 years (Raviglione et al. 2012, WHO 2017). In 2016, there were an estimated 10.4 million new (incident) TB cases worldwide, of which 6.2 million (59\%) were among men, 3.2 million (31\%) among women and 1.0 million (10\%) among children

Date of Submission: August 23, 2018

Published Online: January 2019 and 1.7 million died from the disease (including 0.4 million among people with human immunodeficiency virus) (WHO 2017). Over 95\% of cases and deaths occur in developing countries.

Anti-tuberculosis drug resistance is a major public health problem that threatens progress made in TB care and control worldwide (WHO 2012). Drug resistance arises due to improper use of antibiotics in chemotherapy of drug susceptible TB patients and because of insufficient diagnostic facilities (Zhao et al. 2012). Drug resistant

Date of Acceptance: November 12, 2018

DOI: https:/ / doi.org/10.3126/tujm.v5i0.22314 
bacteria especially MDR-TB persists as a global public health problem (Chiang et al. 2013). MDR-TB is TB due to organism which show high level resistance to both isoniazid (INH) and rifampicin (RIF) with or without resistance to other anti-TB drugs (Ormerod 2005). The emergence of extensively drug resistant tuberculosis (XDR-TB), defined as TB resistant to INH, RIF, quinolones and at least one of three injectable second line drugs (kanamycin, capreomycin or amikacin), in every region of world has raised further alarms about the future or TB control (Marahatta et al. 2010). Globally an estimated $4.1 \%$ of new cases and $19 \%$ of previously treated cases had MDR-TB and estimated 6.2\% patient with MDR-TB had extensively drug resistant TB (XDRTB). WHO estimates that there were 600000 new cases with resistance to rifampicin (RIF), of which 490000 had MDR-TB (WHO 2017).

TB is a major public health problem in Nepal and ranks as one of the most prevalent communicable diseases throughout the country (Upadhyaya et al. 2014). About $45 \%$ of the total population is infected with $\mathrm{TB}$, of which $60 \%$ are adult. Each year about 45000 people develop active TB, out of which 20500 have infectious disease and 5000-7000 people are dying every year by TB (DoHS 2014), 9.3\% of new patient develop resistant to at least one drug and level of MDR-TB among new cases is $2.2 \%$ while among retreatment cases is $17.4 \%$ (NTC 2014).

Early diagnosis of tuberculosis and rapid detection of rifampicin (RIF) and isoniazid (INH) resistant is important for the early administration of appropriate therapeutic agent for the prevention of additional resistance development (Bossier et al. 2006).

\section{MATERIALS AND METHODS}

This cross-sectional study was conducted at National Tuberculosis Centre (NTC), located at Bhaktapur district, Nepal from January to July 2016. One hundred forty-five new or previously treated patients of any age and gender visiting NTC were enrolled in the study. Informed consent was taken from each patient. Early morning sputum samples were collected from patients attending NTC after taking informed consent. Each patient was instructed to collect sputum sample in wide mouth, transparent, sterile, screw capped plastic container (Tille et al. 2014). If the sputum was saliva, blood stained, less than $3 \mathrm{ml}$ and contained greater than 25 epithelial cells per low power field and less than 10 pus when observed microscopically, then the specimen was rejected (Cheesbrough 2002; Lee et al. 2015). Digestion and decontamination of sputum sample was done using sodium hydroxide method (Modified Petroff's method) in order to remove contaminants. The digested specimen was spread evenly in glass slide, air dried, heat fixed and stained by ZN staining and observed under oil immersion objective for the presence of acid fast bacilli (AFB). Approximately, 0.2 $\mathrm{ml}$ of each of resuspended sediment was inoculated to each of duplicate Lowenstein Jensen (LJ) media tubes and incubated at $37^{\circ} \mathrm{C}$. The tubes were slightly opened during incubation for about 2-3 days in order to evaporate excess water and then tightened the cap tightly. The tubes were observed after 24-48 hours to discard contaminated tubes. Then, the tubes were observed weekly up to 8 weeks. M. tuberculosis was identified by observing their growth rate and colony morphology (rough, tough, buff). The colonies on the LJ media were further confirmed by conventional biochemical tests such as susceptibility to para-nitro benzoic acid (PNB), nitrate reductase test, niacin test and heat labile catalase test (RNTCP 2009). Drug susceptibility testing was done by proportion method. For this, one $\mathrm{ml}$ of sterile distilled water (SDW) was added in a sterile glass homogenizer. One loopful of colonies was transferred to a glass homogenizer with six $3 \mathrm{~mm}$ glass beads and vortexed for 20-30 seconds. Four $\mathrm{ml}$ of sterile distilled water was added and homogenized by rotating the inner rotator. Turbidity with McFarland standard No. 1 was adjusted with sterile distilled water and $2 \mathrm{ml}$ loopful of above solution was mixed with $2 \mathrm{ml}$ of sterile distilled water in order to make the $10^{-2}$ dilution (S2). Similarly, two loopful of S2 was added with $2 \mathrm{ml}$ of sterile distilled water in order to make $10^{-4}$ dilution (S4). One loopful each of $10^{-2}$ suspension was inoculated into control tube as well as set of drugs containing media. Similarly, one loopful each of $10^{-4}$ suspension was inoculated into two control tubes as well as set of drugs containing media.The slopes were incubated at $37^{\circ} \mathrm{C}$ and read at 28 days and again in the 42 days (WHO 2001). The obtained data was analyzed using SPSS 16.0 software. The chi square test was used to associate pulmonary tuberculosis with habit of smoking, alcoholism, coughing, travel to foreign country and BCG vaccination. The P-value < 0.05 was considered statistically significant. 


\section{RESULTS}

A total of 145 patients were enrolled in the study. Out of 145 patients, $99(68.2 \%)$ were male. The highest percentage of samples were from $44.1 \%$ age group 20 - 40 years $(44.1 \%)$ followed by age group 40 - 60 years $(37.2 \%)$ and $>60$ years $(13.1 \%)$ while the lowest percentage was seen in age group $<20$ years (5.5\%). Among 145 included patients, 68 (46.9\%) were positive by $\mathrm{ZN}$ microscopy and $72(49.7 \%)$ were culture positive.

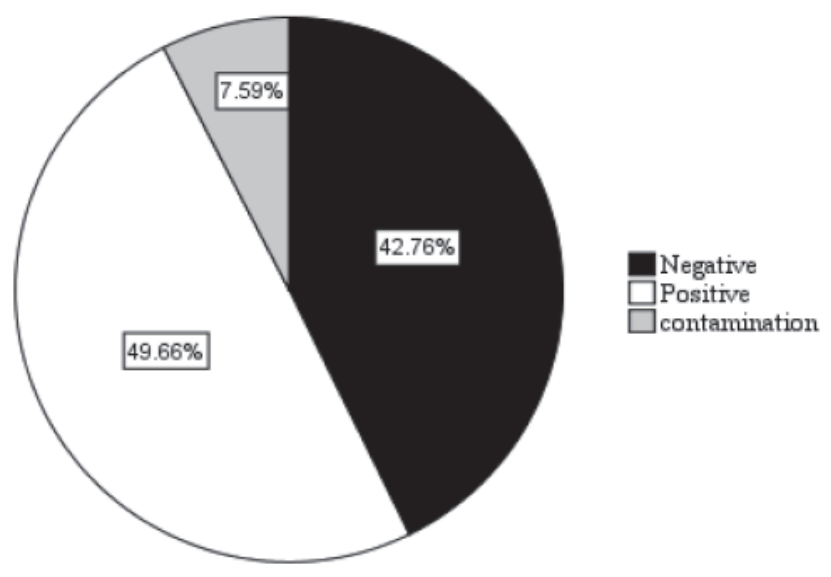

Figure 1: Distribution of culture positive, culture negative and contamination among total specimens

Considering culture as gold standard, the sensitivity and specificity of $\mathrm{ZN}$ staining were found to be $87.5 \%$ and $96.8 \%$ respectively.
Among four anti-tuberculosis drugs, the culture positive isolates $(n=72)$ were found to be most susceptible to EMB and least susceptible to RIF.

Table 1: Distribution of drug resistance among culture positive isolates with four anti-tubercular drugs $(n=72)$

\begin{tabular}{lcc}
\hline Drug & Susceptible N (\%) & Resistant N (\%) \\
\hline RIF & $57(79.1 \%)$ & $15(20.8 \%)$ \\
INH & $58(80.5 \%)$ & $14(19.4 \%)$ \\
STR & $61(84.7 \%)$ & $11(15.2 \%)$ \\
EMB & $70(97.2 \%)$ & $2(2.7 \%)$ \\
\hline
\end{tabular}

$\mathrm{INH}=$ isoniazid; $\mathrm{RIF}=$ rifampicin; $\mathrm{STR}=$ streptomycin; $\mathrm{EMB}=$ ethambutol

Among 72 culture positive isolates of MTB responsible for PTB, $25 \%(n=18)$ were DR strains resistant to at least one drug of which $6.9 \%(n=5)$ were isolated from new patients and $18.1 \%(n=13)$ were identified from previously treated patients. In this study, it was observed that triple drug resistance was highest 7 (9.7\%) followed by mono drug resistance 5 (6.9\%) and double drug resistance 4
(5.5\%), while quadruple drug resistance was lowest 2 $(2.7 \%)$. Drug resistance pattern among MTB isolates is shown in table 2. The total prevalence of MDRTB was $15.2 \%(n=11)$. Out of 18 resistant isolates, $61.1 \%(n=11)$ were resistant to both RIF and INH, $21.4 \%(n=3)$ resistant to INH were susceptible to RIF and $26.6 \%(n=4)$ resistant to RIF were susceptible to INH. 
Table 2: Distribution of drug resistance pattern among MTB isolates $(\mathrm{n}=72)$

\begin{tabular}{|c|c|c|}
\hline & Resistance to any drugs & 18 (25\%) \\
\hline \multirow{13}{*}{ Drugs } & One drug resistance & \\
\hline & RIF & $1(1.3 \%)$ \\
\hline & INH & $4(5.5 \%)$ \\
\hline & Total & $5(6.9 \%)$ \\
\hline & Two drugs resistance & \\
\hline & $\mathrm{INH}+\mathrm{RIF}$ & $2(2.7 \%)$ \\
\hline & $\mathrm{INH}+\mathrm{STR}$ & $2(2.7 \%)$ \\
\hline & Total & $4(5.5 \%)$ \\
\hline & Three drugs resistance & \\
\hline & $\mathrm{INH}+\mathrm{RIF}+\mathrm{STR}$ & 7 (9.7\%) \\
\hline & Four drugs resistance & \\
\hline & $I N H+R I F+S T R+E M B$ & $2(2.7 \%)$ \\
\hline & MDR - TB & 11 (15.2\%) \\
\hline
\end{tabular}

MTB and alcoholism $(P=0.92)$, BCG vaccination $(P=0.238)$ and travel history to foreign country $(P=0.534)$ were not statistically significant but with the habit of smoking $(P=0.001)$ and coughing $(P=0.009)$ were found statistically significant.

\section{DISCUSSION}

In this study, cases of MTB identified by culture was in consistent with NTC report 2014 but higher than recent study of Maharjan et al. (2017). This finding was lower than in other developing countries like Bangladesh and Iran (Mottalib et al. 2011; Nasiri et al. 2014).The higher incidence of TB among man could be attributed to vulnerability of men to TB because of their mobile life style and exposure to predisposing factors like smoking, alcohol, drug abuse (Bhatta et al. 2009). The present finding was in contrast to previous study in Pakistan in which rates of notified TB cases is higher in young females (Codlin et al. 2011).

The findings of this study showed that majority of the TB patients belong to the economically active young age group of 20-60 years. This finding was consistent with the earlier finding by Bhatt et al. (2009) in which majority of TB patients were in the age group of 2150 years, suggesting that $\mathrm{TB}$ is common among the economically active group having direct impact to the family and the national economy.

Comparison of $\mathrm{ZN}$ staining to the culture, which is regarded as gold standard, in this study showed higher sensitivity and slightly less specificity than previous study (Abdelaziz et al. 2016). The study conducted in African population with a high prevalence of HIV shows acid-fast microscopy was highly sensitive $(93.1 \%)$ and specific (100\%) when performed by trained technologists in a carefully controlled manner using established techniques (Sheay et al. 2009). This finding implies that the $\mathrm{ZN}$ staining is quite efficient in the diagnosis of pulmonary tuberculosis, but RT-PCR showed higher sensitivity and specificity as compared to ZN staining in earlier study (Upadhyaya et al. 2014). This shows tuberculosis diagnosis by PCR is highly efficient than conventional method of diagnosis.

Drug resistant cases detected in this study was less than the research conducted by Thapa et al. (2016) in German-Nepal tuberculosis project laboratory which showed $31.1 \%$ of DR cases. Maharjan et al. (2017) also noted 37.2\% DR cases.The MDR strains prevalence in TB patients in this study was similar to previous studies (Thapa 2016; NTC 2016), but slightly higher than the research conducted in 2011 (7.9\%) and 2015 (11.7\%) (Maharjan et al. 2017; Wagley et al. 2016). This implies MDR strains prevalence in TB patients is continually increasing which poses a serious public health problem due to poor patient management, non-adherence to the prescribed regimen, irregular supply of drugs, poor quality of drugs and poor national TB control programme. INH monoresistance in this study was lower than reported from South eastern China (3.5\%) (Liu et al. 2013), Kenya (12.9\%) (Ndung's et al. 2012) and Mozambique (14.9\%) (Nunes et al. 2008) and RIF monoresistance was higher than INH monoresistance but Rijal et al. (2005), Maharjan et al. (2017) showed 
different results. The probable reason for RIF resistance might be due to broad use of RIF for the treatment of other bacterial infections.

\section{CONCLUSION}

With reference to culture as gold standard; ZN staining is quite efficient in the diagnosis of PTB, which is economically viable in low resource setting countries likeNepal.MDR-TBcases are increasing. The association of MTB infection with smoking and coughing patients were found to be statistically significant.

\section{ACKNOWLEDGEMENTS}

We would like to thank Central Department of Microbiology, Tribhuvan University, Kathmandu, Nepal and National Tuberculosis Centre, Nepal for support as well as patients for providing their samples.

\section{CONFLICT OF INTEREST}

The authors declare no conflict of interest.

\section{REFERENCES}

Abdelaziz MM, Bakr WM, Hussien SM and Amine AE (2016). Diagnosis of pulmonary tuberculosis using Ziehl-Neelsen stain or cold staining techniques? J Egypt Public Health Assoc 91(1):39-43.

Bhatt CP, Bhatt AB and Shrestha B (2009). Nepalese people's knowledge about tuberculosis. SAARC J Tuber Lung Dis HIV/AIDS 6 (2): 31-37.

Brossier F, Veziris N, Truffot-Pernot C, Jarlier V and Sougakoff $W$ (2006). Performance of the genotype MTBDR line probe assay for detection of resistance to rifampin and isoniazid in strains of Mycobacterium tuberculosis with low- and highlevel resistance. J Clin Microbiol 44: 3659-3664.

Cheesbrough M (2002). District laboratory practice in Tropical countries part II. Low price edition, Cambridge University Press, India, 71-76.

Chiang CY, Van Weezenbeek C, Mori T and Enarson DA (2013). Challenges to the global control of tuberculosis. Respirology 18(4): 596-604.

Codlin AJ, Khowaja S, Chen Z, Rahbar MH, Qadeer E, Ara I, McCormick JB, Fisher-Hoch SP and Khan AJ (2011). Gender Differences in Tuberculosis Notification in Pakistan. J Trop Med Hyg 85(3): 514-517.

DoHS (2016). Annual Report 2015/2016. Government of Nepal, Ministry of Health, Department of
Health Services, Kathmandu.

Lee YJ, shin S, Roh EY, Kim DK, Chung HS and Lee CH (2015). Acceptability of Sputum Specimens for Diagnosing Pulmonary Tuberculosis. J Korean Med Sci 30(6): 733-736.

Liu Q, Zhu L, Shao Y, song H, Li G, Zhou Y, Shi J, Zhong C, Cheng C and Lu W (2013). Rates and risk factors for drug resistance tuberculosis in Northeastern China. BMC Pub. Health 13: 1171.

Maharjan S, Singh A, Khadka DK and Aryal M (2017). Drug Resistance Pattern in Pulmonary Tuberculosis Patients and Risk Factors Associated with Multi-Drug Resistant Tuberculosis. Biomedical Life Sci J 5(2): 106-117.

Marahatta SB, Kaewkungwal J, Ramasoota P and Singhasivanon P (2010). Risk factors of Multidrug Resistant Tuberculosis in central Nepal: A pilot study. Kathmandu Uni Med J 9(32):392-397.

NationalTuberculosis Center(NTC) (2017). Government of Nepal, Ministry of Health, Department of Health Services, Kathmandu.

National Tuberculosis Programme Nepal (2016). Annual Report 2072/2073 (2016). Government of Nepal, Ministry of Health \& Population, Department of Health Services, National Tuberculosis Center, Thimi, Bhaktapur.

National Tuberculosis Programme Nepal (2014). Annual Report 2070/2071. Government of Nepal, Ministry of Health \& Population, Department of Health Services, National Tuberculosis Center, Thimi, Bhaktapur.

Ndung'u PW, Kariuki S, Ng'ang'a Z and Revathi G (2012). Resistance patterns of Mycobacterium tuberculosis isolates from pulmonary tuberculosis patients in Nairobi. J Infect Dev Ctries 6(1): 33-39.

Nunes EA, De Capitani EM, Coelho E, Panunto AC, Joaquim OA and Ramos MC (2008). Mycobacterium tuberculosis and nontuberculous mycobacterial isolates among patients with recent HIV infection in Mozambique. J Bras Pneumol 34(10): 822-828.

Ormerod LP (2005). Multi drug-resistant tuberculosis (MDR-TB): epidemiology, prevention and treatment. British Med Bul 73: 17-24.

RNTC Programme (2009). Revised National TB Control 
Programme Training Manual for Mycobacterium tuberculosis Culture \& Drug Susceptibility Testing. Training Manual, Indian Council of Medical Research) and National Tuberculosis Institute, Central TB Division, Nirman Bhawan, New Delhi.

Raviglione M. Marais B, Floyd K, Lonnroth K, Getahun H, Migliori GB, Harries AD, Nunn P, Lienhardt C, Graham S, Chakaya J, Weyer K, Cole S, Kaufmann SH and Zumla A (2012). Scaling up interventions to achieve global tuberculosis control: progress and new developments. Lancet 379(9829): 1902-13.

SAARC TB and HIV/AIDS Centre. SAARC Training Modules for AFB Smear Microscopy and Quality Assurance in AFB Smear Microscopy. Trainer's Manual. SAARC TB and HIV/AIDS Centre, SAARC TB and HIV/AIDS Centre, Thimi, Bhaktapur 2011.

Shea YR, Davis JL, Huang L, Kovacs JA, Masur H, Mulindwa F, Chow Y and Murray PR (2009). High Sensitivity and Specificity of AcidFast Microscopy for Diagnosis of Pulmonary Tuberculosis in an African Population with a High Prevalence of Human Immunodeficiency Virus. J Clin Microbiol 47(5): 1553-1555.

Thapa G, Pant ND, Khatiwada S, Lekhak B and Shrestha B (2016). Drug susceptibility patterns of the Mycobacterium tuberculosis isolated from previously treated and new cases of pulmonary tuberculosis at German-Nepal tuberculosis project laboratory, Kathmandu, Nepal. Antimicro Resis Infect Control 5(1):30.

TillePM(2014). Baileyand Scotts's Diagnostic Microbiology. $13^{\text {th }}$ edition, Mosby Inc, USA, 484-512.

Upadhyaya SK, Dixit SM, Shrestha S, Dangol SD, Pokhrel D, Banjara S, Shrestha KS, Hengoju S and Dahal BK (2013). Assessment of detection of Mycobacterium tuberculosis in sputum samples by Real time PCR based method. J Sci Eng Technol 9(1): 181-188.

Wagley U, Bhatta DR, Rijal KR and Ghimire G (2014). Distribution of Mycobacterium species present in sputum of suspected pulmonary tuberculosis patients. SAARC J Tuber Lung Diseases HIV/AIDS 6(1): 19-23.

WHO. Global tuberculosis report 2017. Geneva, Switzerland 2017.

WHO. Global tuberculosis report 2012. Geneva, Switzerland 2012.

WHO. Guidelines on Standard Operating Procedures for Laboratory Diagnosis of HIV-Opportunistic Infections. Regional Office for South-East Asia, New Delhi 2001.

Zhao P, Li XJ and Zhang SF (2012). Social behavior risk factors for drug resistant tuberculosis in mainland China: a meta-analysis. J Int Med Res 40(2): 436445. 